\title{
NUEVA ESPECIE DEL GENERO ESTOLA FAIRMAIRE ET GERMAIN 1859, PARA CHILE (COLEOPTERA: CERAMBYCIDAE, DESMIPHORINI)
}

\author{
A NEW SPECIES OF THE GENUS ESTOLA FAIRMAIRE ET GERMAIN 1859, \\ FROM CHILE (COLEOPTERA: CERAMBYCIDAE, DESMIPHORINI)
}

\author{
Juan Barriga ${ }^{1}$, Tomás Moore ${ }^{2} \&$ Danilo Cepeda ${ }^{3}$ \\ 'Universidad Católica del Maule, Casilla 139, Curicó, Chile; Email: colneotrop@yahoo.com. \\ ${ }^{2}$ El Vergel 2245. Departamento. 28 D, Providencia, Santiago, Chile. tmoore@vtr.net \\ ${ }^{3}$ Museo Entomológico, Luis Peña, Departamento de Sanidad Vegetal, Facultad de Ciencias Agronómicas, \\ Universidad de Chile. Santiago, Chile. Email: museoent@uchile.cl; Casilla 1004.
}

\begin{abstract}
RESUMEN
Se describe una nueva especie del género Estola para Chile, basado esencialmente en las figuras de genitalia masculina y femenina. Esta especie, que ataca la Araucaria araucana, se diferencia de las otras dos especies chilenas del género particularmente por su tamaño y por la forma de la espina del pronoto.

Palabras claves: Taxonomía, Cerambycidae, Lamiinae, Desmiphoriini, Estola kuscheli n. sp.
\end{abstract}

\section{ABSTRACT}

A new species of Estola living on dead Araucaria araucana wood from Chile is described. Description is mainly based on male and female genitalia and external features. This species is differentiated from the two other species of the genus, particularly by its size and the shape of pronotal spines.

Keywords: Taxonomy, Cerambycidae, Lamiinae, Desmiphoriini, Estola kuscheli nov.sp.

\section{INTRODUCCION}

El género Estola fue creado por Fairmaire et Germain (1859) a partir de la separación del género Hebestola Blanchard, debido a la existencia de caracteres disímiles, estableciéndose dos nuevas especies propias de Chile, E. hirsuta y E. unicolor. Con posterioridad Aurivillius (1922) resuelve un problema de nomenclatura para $E$. hirsuta, denominándola $E$. hirsutella, nombre con el cual figura en el catálogo de Blackwelder (1946), perteneciente a la tribu Estolini.

Cerda (1986) sólo reconoce la especie E. hirsutella, mencionando a $E$. unicolor en nomina dubia; adscribe esta especie a la tribu Ptericoptini junto al gé- nero Bisaltes Thomson, entregando citas referentes a su distribución geográfica.

En relación a referencias de hospederos, Barriga et al. (1993) citan a $E$. hirsutella como especie xilófaga de madera muerta de Araucaria araucana (Araucariaceae).

En reciente catálogo Monné \& Giesbert (1995) incluyen el género en la tribu Desmiphorini. Estola cuenta con un total de 88 especies, esencialmente de distribución sudamericana, citando a $E$. hirsutella y E. unicolor sólo para Chile.

El siguiente aporte tiene por objetivo dar a conocer una nueva especie del género, Estola kuscheli, entregando descripción e ilustraciones de genitalias masculina y femenina. 


\section{MATERIALES Y METODOS}

Los estudios morfológicos de la nueva especie se realizaron en base a 253 ejemplares provenientes de las siguientes colecciones: Colección del primer autor (JEBC); Colección del Museo Entomológico Luis Peña del Departamento de Sanidad Vegetal de la Universidad de Chile (MEUC); Colección del Museo Nacional de Historia Natural, Santiago, Chile (MNNC); y a las siguientes colecciones particulares, de los señores: Tomas Moore (TPMR); Pedro Vidal (PVGH); José Mondaca (JMEC); V. Manuel Diéguez (VMDM); Sergio Roitman (SRRC); Tomás Fichet (TFCC) y Tomislav Curkovic(TCCC).

Las observaciones de los adultos se realizaron en lupa estereoscópica Leitz 12,5x y las de estructuras de la genitalias, en microscopio de fase contrastada Leitz.

Para estudio de las estructuras de las genitalias se trató con método sugerido por Barriga \& Cepeda (2004). Las preparaciones fueron depositadas en la colección del MEUC.

\section{RESULTADOS}

DESCRIPCIÓN

DiAgnosis: Especie pequeña, cuerpo de color general marrón oscuro, vista dorsal abundantemente piloso; antenas de tonalidad clara sin áreas pilosas en la base; pronoto y élitros con pilosidad larga de color rubio blanquecina y tumbada, con mayor densidad en márgenes, la pilosidad de la superficie elitral se muestra como manchas aisladas, con algunos largos pelos erizados y dispersos. Espina de los márgenes del pronoto larga y aguda; escutelo piloso; ventralmente el abdomen presenta pubescencia blanquecina y entremezclada. Largo promedio: hembras $3,8 \mathrm{~mm}$; machos $3,5 \mathrm{~mm}$; ancho humeral: hembras: $1,2 \mathrm{~mm}$; machos $1,0 \mathrm{~mm}(\mathrm{n}=253)$.

\section{Estola kuscheli n. sp.}

Holotipo macho (Fig. 5)

CABEZA: Transversa, subigual en ancho al pronoto en su margen anterior, cubierta de pilosidad rubia blanquecina con mayor densidad alrededor de los ojos, sutura longitudinal en la cabeza presente y marcada desde el occipucio hasta el margen del clípeo, frente ancha y plana entre los ojos, los cuales son muy escotados y groseramente facetados, labro pequeño y redondeado. Antenas sobrepasan apenas el largo del cuerpo, de tonalidad clara, primer antenito alargado, grueso y robusto, segundo corto y un tercio del largo del primero, tercero y cuarto subiguales, quinto al undecimo filiformes recubiertos en toda su extensión de fina pilosidad rubia blanquecina, con algunos pocos pelos erizados y erectos.

PRONOTO: Ligeramente más ancho que largo, de margen anterior y posterior subiguales, recubiertos de pilosidad rubia blanquecina especialmente en sus bordes, espina en margen lateral central larga y aguda, ligeramente levantada y pilosa. El disco finamente punteado, proceso prosternal no más ancho que la cavidad coxal, de forma triangular, reticulado y de lados ensanchados.

ELITROS: Más ancho que el pronoto, tres veces su largo; ángulos humerales ampliamente prominentes, lados paralelos y ápices redondeados, de superficie convexa, recubiertos de abundante pilosidad rubia blanquecina, especialmente en los márgenes, semejando manchas aisladas, así como de largos pelos erizados, esparcidos, puntuación redondeada y marcada, densa y profunda. Escutelo ancho redondeado y piloso.

Abdomen: Finamente punteado y cubierto de abundante pilosidad rubia blanquecina tumbada, último esternito ampliamente redondeado, de margen posterior subtruncado, convexo y más corto que el precedente.

Patas: Con tercio distal de las tibias con largos pelos erectos.

Holotipo macho, Chile, prov. Malleco, Nahuelbuta, 2/02/1994, leg. J. E. Barriga, depositado en la colección del primer autor (JEBC) junto a 126 paratipos. Largo: 3,5 mm; ancho humeral: 1,2 $\mathrm{mm}$ Alotipo hembra (Fig. 6), Chile, prov. Arauco, Nahuelbuta, enero 1989, leg. J. E. Barriga, similar al holotipo excepto en el largo de las antenas las cuales son ligeramente más cortas que el cuerpo, y el último esternito que es más de dos veces el largo que el precedente, depositado junto a 5 paratipos en la colección del Museo Entomológico Luis Peña de la Facultad de Ciencias Agronómicas, de la Universidad de Chile. (MEUC). Largo: $4 \mathrm{~mm}$; ancho humeral: $1,4 \mathrm{~mm}$. 
Distribución Geográfica: Chile, provincia de Malleco, en las siguientes localidades: Nahuelbuta, Pichinahuel, Cuesta Las Raíces, Tolhuaca, Termas de Río Blanco, Malalcahuello, Lonquimay; provincia de Cautín: P. N. Conguillío. Considerando el grado de asociación de esta nueva especie a las araucarias, es posible que su distribución se extienda hacia Argentina.

HosPedero: Araucaria araucana (Araucariaceae) Genitalia masculina y femenina: Aedeagus (Fig.1) de ápice agudo, saco interno con porción distal con procesos espiniformes; tegmen de porción anterior lobulada y con largas setas distales.

Ovipositor (Fig.2) amplio y quitinoso, con estilos ovoides; espermateca (Fig.3) sinuosa en forma de "u"; $8^{\circ}$ tergito (Fig.4) amplio, ancho y de margen redondeado, recubierto de fina pilosidad.

VARIACIONES INTRAESPECíficas: En todos los ejemplares observados, la especie es constante en sus caracteres morfológicos. El largo de las antenas no corresponde a un parámetro o característica sexual.

Comentarios: Tomando en consideración que el taxón cuenta con un gran número de especies, cabe destacar que el género fue creado a partir de entidades chilenas, las cuales están asociadas a la Araucaria araucana. Motivo por el cual hemos estimado que este taxón contiene correctamente a esta nueva especie.

La nueva especie se diferencia de E. hirsutella fundamentalmente por los siguientes caracteres: su tamaño ostensiblemente menor, pilosidad y colorido, falta de banda clara en la base de los antenitos y la espina del margen del pronoto más aguda.

Ante la imposibilidad de contar con ejemplares de la especie E. unicolor correctamente determinada, nos basamos en la descripción original.

E. unicolor Long.7 mill. Oblonga, brunnea pube cinereo-rufescente sat dense tecta, pilis griseis rarius hirsuta; antennis corpore brevioribus; prothorace convexiore minus dense punctato, utrinque spina brevísima arcuato; elytris oblongis, apicem versus leviter attenuatis, punctatosubstriatis, interstitiis suturam versus leviter elevatis, apice oblique subtruncatis

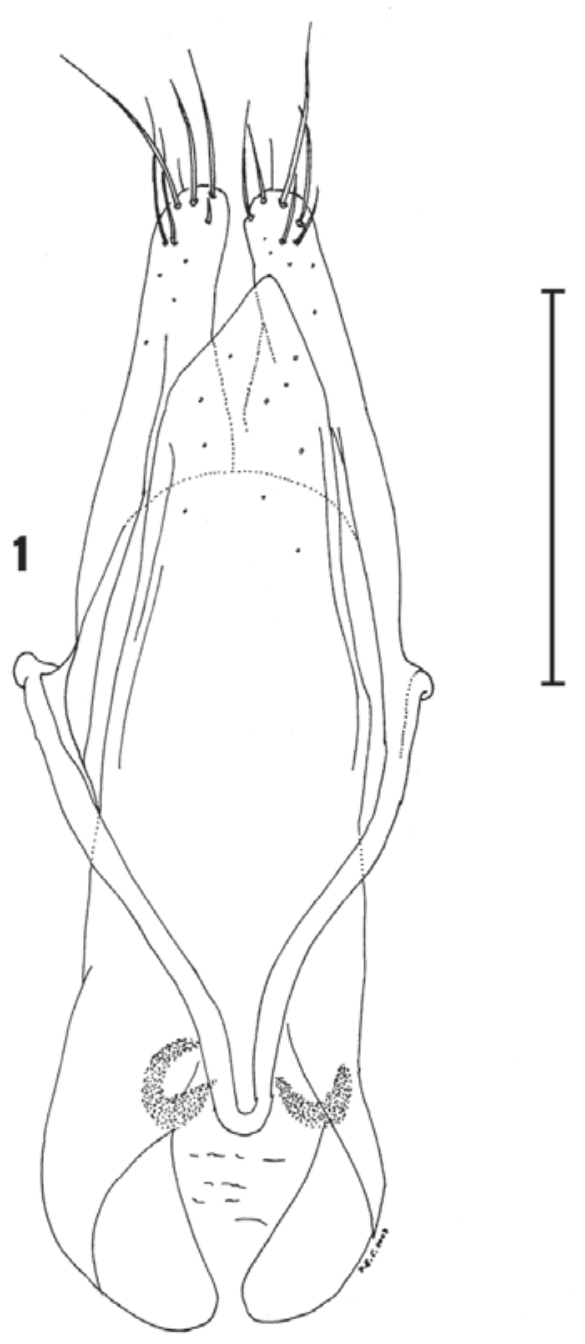

FIGURA 1. Estola kuscheli n. sp. : tegmen y aedagus. Escala: $0.25 \mathrm{~mm}$.'

Figure 1. Estola kuscheli n. sp. : tegmen and aedeagus. Scale: $0.25 \mathrm{~mm}$.

Para poder diferenciar a E. kuscheli de E. unicolor destacamos los siguientes caracteres: su tamaño considerablemente menor, el mayor largo de las antenas, la cabeza con presencia de sutura longitudinal y la puntuación elitral no estriada ni elevada.

Etimología: Esta especie está dedicada al Entomólogo Dr. G. Kuschel, quien fuera el primer colector. 
Gayana 69(2), 2005
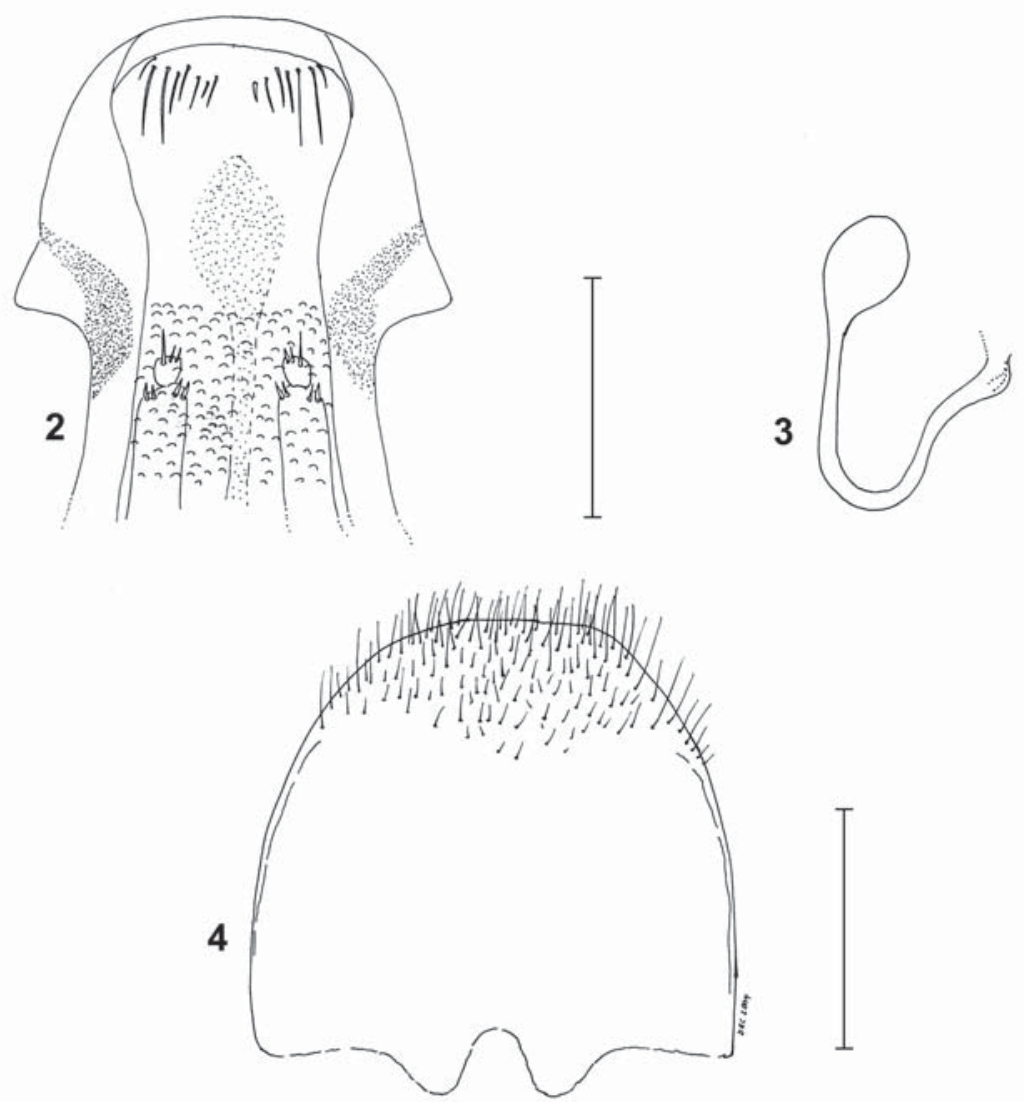

FiguRAs 2-4. Estola kuscheli n. sp.: 2. Detalle ovipositor, 3. Espermateca ,4. $8^{\circ}$ tergito. Escala: 0.5 mm.

Figures 2-4. Estola kuscheli n. sp.: 2. Detail of ovipositor, 3. Espermatheca, 4. $8^{\circ}$ tergite. Scale: $0.5 \mathrm{~mm}$.
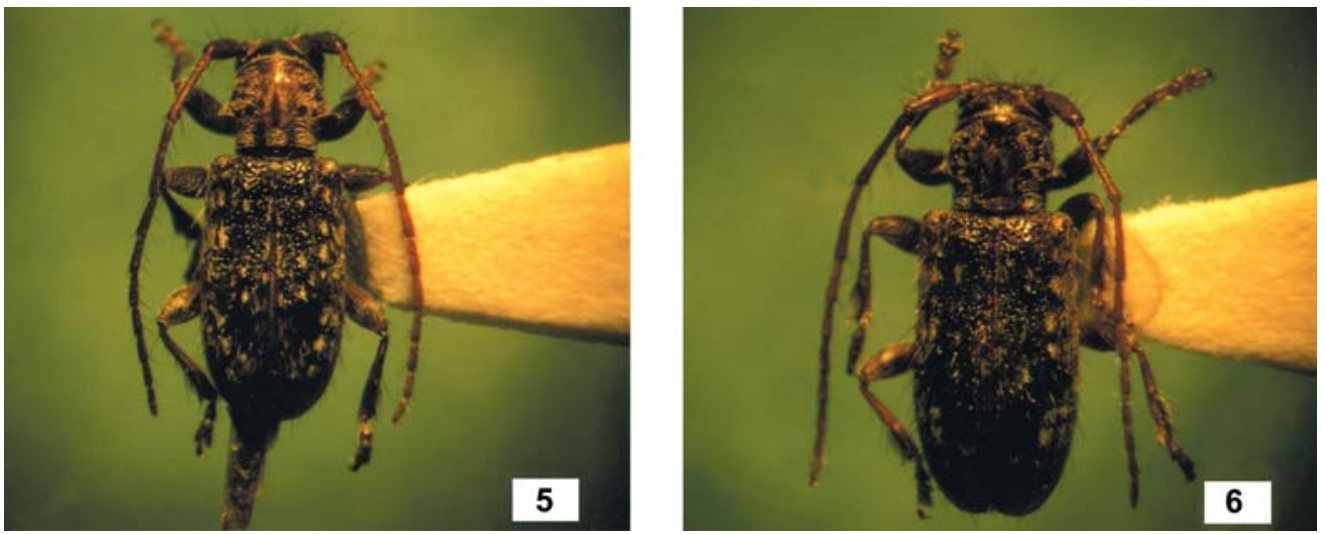

FIGURAS 5-6. Estola kuscheli n. sp.: 5 holotipo macho adulto, vista dorsal, 6 alotipo hembra adulto vista dorsal.

Figures 5-6. Estola kuscheli n. sp.: 5 dorsal view of adult male holotype, 6 dorsal view of adult female allotype. 
MATERIAl EXAminado: 253 ejemplares

Chile, provincia de Malleco: Cuesta Las Raíces, 1621/02/2001, leg.V. M. Diéguez; igual localidad, 4, 10, 13, 23/02/2001, leg. P. Vidal; igual localidad, 13/02/ 1999, coll. M. Guerrero; igual localidad, 21/02/2001, leg. A. Soto; igual localidad 9/02/2000, leg. C. Rojas; igual localidad, 17, 22/02/2001 coll. T.Moore; Termas de Río Blanco, enero 1997, coll. J. Mondaca; Tolhuaca, 17, 19/02/1996, coll. J. Mondaca; igual localidad, 10/02/2001, leg. P. Vidal; Nahuelbuta, $9^{\text {a }}$ Región, 20/01/1993, coll. T. Curkovic; P. N. Nahuelbuta, Pichinahuel, 22/01/2002, coll. T. Moore; igual localidad, $1.200 \mathrm{~m}, 25 / 11 / 2004,12 / 02 / 2005$, leg. J. E. Barriga, foggin s/Araucaria, S $37^{\circ} 47^{\prime} \mathrm{W} 73^{\circ}$, Nahuelbuta, 18-22/01/1993, leg. T. Fichet; vn. Lonquimay 1200-1400 ms.n.m.22-24/02/2003, leg. T. Fichet; P.N. Nahuelbuta, 24/01/2001, leg. J. E. Barriga; igual localiadad, 24/01/1985, coll. S.Roitman; Malalcahuello, IX Reg. 9/02/2000, leg. C.Rojas; Angol, Nahuelbuta, Coimallin, sobre Baccharis, 24 ene. 1985, col. S. Roitman. Provincia de Cautín: P. N. Los Paraguas, 20/02/1996, coll. J. Mondaca; igual localidad, 20 feb.1982, col. M Elgueta; Conguillío, Chile, IX Región 22, 25/02/2001, Coll. T. Moore.

Depósito de paratipos: En las siguientes colecciones: Museo Nacional de Historia Natural, Santiago, Chile (MNNC) (15 ej.); Museo de Zoología de la Universidad de Concepción (UCCC) (5 ej.); colección del señor T. Moore (TPMR) (7 ej.); Instituto de Entomología, J. Herrera, Universidad Metropolitana de Ciencias de la Educación, Santiago, Chile (IEUMCE) (2 ej.); colección Universidad de Valdivia (CFVA) (2 ej.); Laboratorio de Entomología, Universidad de La Serena, La Serena, Chile (LEULS) (3 ej.); colección del Museo de la Plata, Argentina (MLPA) (2 ej.); colección del Museo Argentino de Ciencias Naturales (MACN) (2 ej.) colección del Instituto Miguel Lillo, Tucumán, Argentina (2 ej.); Museo de Zoología Universidad de Sao Paulo (MZSP) (2 ej.); Musée Nationale d'Historie Naturelle, Paris-Francia (MNHN) (1 ej.); the Natural History Museum, London, England (BMNH) (1 ej.); Carnegie Museum of Natural History, Pitts- burgh, PA, USA (CMNH) (1 ej.); California Academy of Sciences, San Francisco, CA, USA (CMNC) (1 ej.); Field Museum of Natural History, Chicago, IL, USA (FMNH) (1 ej.) United States Nacional Museum, Washington DC, USA (USNM) (1 ej.); University of Florida Museum of Natural History, Florida USA (FSMC) (1 ej.); New Zealand Auckland, Arthropod collection (NZAC) (1 ej.); CSIRO Australian National Insect collection (ANIC) (1ej.) y en las colecciones privadas: (PVGH) (40 ej.), (TFCC) (4 ej.), (JMEC) (8 ej.), (TCCC) (2 ej.), (VMDM) (11 ej.) y señor Sergio Roitman (5 ej.)

\section{AGRADECIMIENTOS}

Al doctor R. H. González por la lectura crítica de este trabajo y a todos quienes nos facilitaron material para nuestro estudio.

\section{BIBLIOGRAFIA}

Aurivillius, C.1922. Coleopterorum Catalogus, pars.73, Lamiinae, 23:1-322.

Barriga, J. E. \& Cepeda, D. E. 2004. Nuevo género y especie de Compsocerini de Chile (Coleoptera: Cerambycidae). Revista Chilena de Entomología 30 (2): 5-8.

Barriga, J. E., T. Curkovic, T. Fichet, J.L. Henríquez \& J. MACAYA. 1993.Nuevos antecedentes de coleópteros xilófagos y plantas hospederos en Chile, con una recopilación de citas previas. Rev. Chilena Ent., 20:65-91.

BlaCKWELDER, R. E.1946.Checklist of the coleopterous insects of México, Central America, West Indies and South America. Bull. U.S. Nat. Mus., 185 (4):551-763.

Cerda, M. 1986. Lista sistemática de los Cerambycidae chilenos (Coleoptera: Cerambycidae). Rev. Chilena Ent., 14:29-39.

Fairmaire, L. et Germain, Ph. 1859. Revision des coleóptères du Chili. Ann. Soc. Ent. France, 7: 483-532.

Monné, M.A \& Giesbert E.F. 1995 Checklist of the Cerambycidae and Disteniidae (Coleoptera) of the Western Hemisphere. Ed. Wolfsgarden Books, Burbank, California, XIV, 409 pp.

Fecha de recepción: 05/03/05

Fecha de aceptación: 12/08/05 